\title{
Cardiac Rehabilitation of Patients with Acute
} ST-Elevation Myocardial Infarction Undergoing Primary Percutaneous Coronary Intervention in a Han Population in Northern China: A Prospective Cohort Study

\author{
Jingru $\mathrm{Ma} \mathbb{D}^{\prime}$ \\ Yuling Tai ${ }^{2}$ \\ Mengmeng $\mathrm{Fan}^{3}$ \\ Zhansheng Wang ${ }^{4}$ \\ 'Department of Cardiology, The Second \\ Affiliated Hospital, Shenyang Medical \\ College, Shenyang, I I0035, People's \\ Republic of China; ${ }^{2}$ Department of \\ Cardiology, Tongliao City Hospital, \\ Tongliao, 028000, People's Republic of \\ China; ${ }^{3}$ Department of Cardiology, \\ Xinxiang Central Hospital, Xinxiang, \\ 453000, People's Republic of China; \\ ${ }^{4}$ Department of Cardiology, Fourth \\ People's Hospital of Shenyang, Shenyang, \\ I 10034, People's Republic of China
}

Correspondence: Jingru Ma The Second Affiliated Hospital of Shenyang Medical College, No. 64 Qishan West Road, Huanggu District, Shenyang, I 10035, Liaoning Province, People's Republic of China

Tel + 86-18002453999

Email majingru00@126.com
Background: Cardiac rehabilitation (CR) has been associated with improved cardiac function in cardiovascular diseases. Our aim was to explore the factors associated with cardiac function and CR.

Methods: This prospective cohort study had 473 STEMI patients admitted for primary percutaneous coronary intervention $(\mathrm{PCI})$ who were divided into a $\mathrm{CR}$ group (group $\mathrm{A}, \mathrm{n}=$ 104) and a non-CR group (group $B, n=369$ ) based on whether they could complete CR. Patients' clinical features, such as age, hyperlipidemia, family history of premature coronary heart disease (FHPCHD), smoking history, body mass index (BMI, $\mathrm{kg} / \mathrm{m}^{2}$ ), number of diseased vessels, arrhythmia during PCI, N-terminal pro-B-type natriuretic peptide (NTproBNP), left ventricular ejection fraction (LVEF) and major adverse cardiac events (MACEs) at 6 months after PCI were compared. Then, the clinical characteristics of patients were further analyzed according to those with MACEs $(n=78)$ and those without MACEs $(n$ $=395$ ).

Results: After CR of 6 months, NT-proBNP levels ( $\mathrm{p}=0.027), 6-\mathrm{MWD}$ (meter, $\mathrm{P}=0.000$ ), LVEF ( $\mathrm{P}=0.000)$ were significantly improved in group A compared to group $\mathrm{B}$, but not for SMWA $(\mathrm{P}=0.875)$. Multivariate analysis indicated that even though patients in group A (OR $3.06,95 \%$ CI $1.132-8.274, \mathrm{p}=0.03$ ) have a higher incidence of hyperlipidemia, their MACEs (OR 0.191, 95\% CI 0.038-0.961, p =0.05) at 6 months were significantly lower than in group B, mainly because the average patient low age $(<65$ years, OR $0.917,95 \% \mathrm{CI}$ $0.859-0.979, \mathrm{p}=0.01)$ and significant improvement of 6-MWD (OR 7.999, 95\% CI 4.342$14.737, \mathrm{P}=0.00$ ) and the LVEF at 6 months (OR 1.112, 95\% CI 1.072-1.154, $\mathrm{p}=0.00$ ). Further analysis based on the MACES outcomes showed that there were 6 factors associated with the occurrence of MACEs, they were age $>65$ years (OR 1.032, 95\% CI 1.009-1.009, $\mathrm{p}=0.007$ ), smoking history (OR $0.485,95 \%$ CI $0.238-0.989, \mathrm{p}=0.046$ ), education level (OR 2.646, 95\% CI 1.370-5.108, p = 0.004), 6-MWD (OR 1.688, 95\% CI 1.104-2.811, p = 0.044), LVEF (OR 0.958, 95\% CI 0.926-0.991, p = 0.013) and CR (OR 6.271, 95\% CI 2.236-17.590, $\mathrm{p}=0.000)$.

Conclusion: $\mathrm{CR}$, including exercise rehabilitation, is a beneficial option to reduce MACEs in STEMI patients treated with primary PCI.

Keywords: rehabilitation, ST-elevation myocardial infarction, percutaneous coronary intervention, major adverse cardiac events 


\section{Introduction}

Diseases of the cardiovascular system, including acute myocardial infarction (AMI), can result in high rates of disability and death and place a significant burden on society. ${ }^{1}$ Among them, acute ST-segment elevation myocardial infarction (STEMI) is a serious global critical illness and a challenge to human health. Percutaneous coronary intervention (PCI) has become the treatment strategy of choice for reperfusion in STEMI patients. ${ }^{2}$ Among different reperfusion strategies for STEMI, primary PCI was associated with the lowest risk of mortality. ${ }^{3}$ There are approximately 750,000 patients in China underwent PCI treatment in $2017 .^{4}$ In patients with STEMI, PCI technology may relieve atherosclerotic vascular narrowing or thromboembolization, but exercise tolerance may still decline after revascularization. ${ }^{5,6}$ Recent evidence has demonstrated exercise-based cardiac rehabilitation is beneficial in improving clinical outcomes and secondary prevention of acute myocardial infarction. ${ }^{7}$ Comprehensive rehabilitation treatment is related to improved cardiac function and exercise tolerance in cardiovascular diseases.

The values of cardiac rehabilitation (CR) for patients with heart diseases such as coronary artery bypass surgery, heart valve repair, PCI, myocardial infarction (MI), stable angina, stable chronic heart failure, heart transplantation, arrhythmias, and severe arterial hypertension have been demonstrated. ${ }^{8}$ The goals of CR include improvement in exercise tolerance and optimization of coronary risk factors, including improvement in lipid and lipoprotein profiles, body weight, blood glucose levels, blood pressure levels, and smoking cessation. ${ }^{9}$

It has been reported that Asian patients are less involved in CR after PCI than in Western countries. ${ }^{10}$ In a prospective study in Singapore, the participation rate in CR was only $12.3 \%$ among 414 eligible patients. ${ }^{11}$ However, there is an increasing number of STEMI patients after primary PCI who receive $\mathrm{CR}$ including exercise rehabilitation in China. CR not only improves the quality of life of patients but also improves several important outcomes, such as control of cardiovascular diseaserelated risk factors, exercise ability, readmission rates, and mortality. ${ }^{12}$

Our objective was to investigate the relationship between $\mathrm{CR}$ and major adverse cardiac events (MACEs) in STEMI patients after PCI. Data from China may provide further insight into $\mathrm{CR}$ as well as explore factors related to cardiac function and $\mathrm{CR}$.

\section{Methods}

\section{Study Population and General Baseline Characteristics}

Four hundred and seventy-three STEMI patients admitted to the Second Affiliated Hospital of Shenyang Medical College between January 2017 and December 2019 were used as study subjects. Inclusion criteria: Han Chinese diagnosed with STEMI and treated with primary PCI with drug-eluting stents who have been taking Dual antiplatelet therapy (DAPT, clopidogrel $75 \mathrm{mg}$ and aspirin $100 \mathrm{mg}$ daily) postoperatively. Exclusion criteria: (a) cerebrovascular diseases, severe valvular diseases, uncontrolled hypertension or uncontrolled arrhythmia, hypertrophic obstructive cardiomyopathy, aortic dissection, severe orthopedic or neurological disability to exercise, (b) advanced heart failure (functional class IV), (c) individuals with incomplete clinical information who did not undergo enrollment and withdrawal.

General baseline characteristics, blood biochemical indicators including creatine kinase myocardial band (CK$\mathrm{MB})$, Troponin $\mathrm{T}(\mathrm{TnT}), \mathrm{N}$-terminal pro-B-type natriuretic peptide (NT-proBNP) and serum creatinine were obtained from peripheral venous blood within 24 hours of admission for all studied participants. Clinically relevant data included the pre-hospital delay time, door-to-balloon time, the number of diseased-vessels, and the color flow Doppler echocardiography data including left ventricular ejection fraction (LVEF), 6-minute walking distance (6-MWD) in meters, segmental wall motion abnormalities (SWMA) and complications.

\section{Intervention}

In the absence of medical contraindications, the basic medications, including DAPT (dual antiplatelet therapy), ARB (angiotensin receptor blockers), ACEI (angiotensinconverting enzyme inhibitors) B-receptor blocker or statins, etc., were routinely given to patients with STEMI after PCI. The patients were divided into $\mathrm{CR}$ group (group A, n=104) and non-CR group (group B, n=369) according to their wishes and whether they could complete CR. Group A was given the $\mathrm{CR}+$ basic medication therapy. Group B was given the basic medication therapy.

$\mathrm{CR}$ was defined as a combination of cardiac rehabilitation in the acute phase (beginning in the hospital) and the 
convalescence phase (6 months after discharge). CR including exercise rehabilitation was carried out after vital signs stabilized. Patients in group A started CR on the tenth day on average. Exercise rehabilitation was formulated according to the consensus of Chinese experts on exercise rehabilitation after PCI. ${ }^{13}$ Step 1 consisted of low levels of aerobic exercise for 5-10 min. Step 2 consisted of aerobic, resistance, and flexibility exercise lasting 30 $90 \mathrm{~min}$. Step 3 was a relaxation exercise lasting 5-10 min.

The points to note during rehabilitation process were as follows: (a) patients need to be informed about the purpose, methods and warning symptoms of exercise prescription before exercise rehabilitation, (b) patients undergoing early rehabilitation should be evaluated prerehabilitation and post-rehabilitation, (c) cardiac first aid emergency plans, equipment and medicines need to be prepared for the patients with STEMI after PCI during hospitalization, (d) cardiac first aid training for CR doctors and nurses should be conducted regularly. In addition, we further analyzed the potential factors contributing to the occurrence of MACEs based on patients presenting with MACEs $(n=78)$ and those without MACEs $(n=395)$. The written informed consent for $\mathrm{CR}$ was given by all patients.

\section{Follow-Up}

At 6-month follow-up, patients were evaluated and recorded for post-PCI arrhythmia and complications, 6-MWD, NT-proBNP, LVEF, SWMA and MACEs (including death, recurrent myocardial infarction, stroke, and target-vessel revascularization).

\section{Statistical Analysis}

All data were analyzed by IBM SPSS 24.0 (IBM Co., Armonk, NY, USA). Continuous variables in accordance with the normal distribution were presented as mean \pm standard deviation, and the $t$-tests were used to compare the between-group data. Categorical data were presented as percentage (\%) and chi-square tests were used to compare the between-group data. ANCOVA (Analysis of Covariance), followed by Bonferroni test, was performed for NT-proBNP level, 6-MWD, LVEF and SWMA before and after CR. A multivariate logistic regression analysis, using CR or MACEs as the dependent variable (dichotomous for logistic regression) and using age, gender, hyperlipidemia, FHPCHD, smoking history, higher education, BMI $(\mathrm{kg} / \mathrm{m} 2)$, heart rate, number of diseased vessels, arrhythmia during PCI, NT-proBNP, 6-MWD (meter), LVEF (\%), NT-proBNP at 6-month, 6-MWD (meter) at 6-month, LVEF (\%), SWMA and MACEs at 6-month follow-up or $\mathrm{CR}$ as the independent variables, was performed. A $\mathrm{p}$ value $<0.05$ was considered to be statistically significant.

\section{Results}

\section{Baseline Characteristics}

The baseline characteristics of the two groups are shown in Table 1. Age, gender, hyperlipidemia rate, family history of premature coronary heart disease (FHPCHD), rate of smoking history, education levels, body mass index (BMI), heart rate, number of diseased vessels, arrhythmia during PCI, and MACEs at 6-month between the two groups were significantly different (Table $1, \mathrm{P}<0.05$ ).

Among the 620 patients with STEMI, the overall inhospital mortality rate was $3.87 \%$. Among the 473 STEMI patients who underwent primary PCI, pre-hospital delay time and door-to-balloon time averaged 3.66 to 4.46 hours $(\mathrm{P}=0.16)$ and 91.26 to 95.27 minutes $(\mathrm{P}=0.645)$ in groups $\mathrm{A}$ and $\mathrm{B}$, respectively. The overall proportion of comprehensive rehabilitation was $22.0 \%$.

The median duration of follow up was 6 months. 473 (99.4\%) completed 6-month follow-up. The rate of MACEs at 6-month follow-up was significantly lower in group A than in group B $(3.8 \%$ vs $20.1 \%, \mathrm{P}<0.05)$. The proportion of recurrent angina at 6-month follow was significantly lower up in group A than in group B $(0 \%$ vs $11.4 \%, \mathrm{P}<0.05)$. There was no significant difference in the proportion of recurrent myocardial infarction between the two groups at 6-month follow-up (1.9\% vs $1.9 \%$, $\mathrm{P}>0.05)$.

\section{Analysis of Covariance (ANCOVA) Between the Groups}

After 6 months of CR, NT-proBNP levels $(\mathrm{P}=0.027)$, 6-MWD $(\mathrm{P}=0.000)$, and LVEF $(\mathrm{P}=0.000)$ were significantly improved in group A compared to group $\mathrm{B}$, but not for SMWA (Table 2, $\mathrm{P}=0.875$ ).

\section{Multivariate Logistic Regression Analysis} Multivariate analysis for CR (Table 3) showed that although the incidence of hyperlipidemia in group A (OR $3.06,95 \%$ CI 1.132-8.274, $\mathrm{p}=0.03$ ) was higher than that in group B, their MACEs (OR 0.191, 95\% CI 0.038-0.961, $\mathrm{p}=0.05$ ) were significantly lower than that of group $B$ at 6 months, mainly due to the lower average age of patients in group A (<65 years, OR 0.917, 95\% CI 0.859-0.979, 
Table I Baseline Characteristics Between the Two Groups

\begin{tabular}{|c|c|c|c|c|}
\hline Variables & Group A $(n=104)$ & Group B $(n=369)$ & $\mathbf{t} / \chi^{2}$ & $P$ value \\
\hline Age, y & $50.39 \pm 9.381$ & $65.6 \pm 10.752$ & 13.086 & 0.000 \\
\hline \multicolumn{5}{|l|}{ Gender, n (\%) } \\
\hline Male & $90(86.5 \%)$ & 274 (74.3\%) & 6.903 & 0.009 \\
\hline Female & $14(13.5 \%)$ & $95(25.7 \%)$ & & \\
\hline History of hypertension, $\mathrm{n}(\%)$ & $64(61.5 \%)$ & $250(67.8 \%)$ & 1.403 & 0.236 \\
\hline History of diabetes, $\mathrm{n}(\%)$ & $19(18.3 \%)$ & 97 (26.3\%) & 2.818 & 0.093 \\
\hline Hyperlipidemia, n (\%) & $59(56.7 \%)$ & 155 (42.0\%) & 7.101 & 0.008 \\
\hline FHPCHD, n (\%) & $13(12.5 \%)$ & 7 (1.9\%) & 19.982 & 0.000 \\
\hline HMD, n (\%) & $3(2.9 \%)$ & $12(3.3 \%)$ & 0.000 & 1.000 \\
\hline Smoking history, n (\%) & $35(33.7 \%)$ & $75(20.3 \%)$ & 8.076 & 0.004 \\
\hline Higher education, n (\%) & 95 (91.3\%) & $65(17.6 \%)$ & 197.041 & 0.000 \\
\hline BMI, kg/m2 & $27.4 I \pm 2.3 I$ & $25.77 \pm 2.38$ & -5.838 & 0.000 \\
\hline Heart rate, bpm & $76.65 \pm 17.01$ & $72.63 \pm 17.13$ & 2.061 & 0.040 \\
\hline Serum creatinine, $\mu \mathrm{mol} / \mathrm{L}$ & $73.32 \pm 26.95$ & $77.05 \pm 24.52$ & -1.339 & 0.181 \\
\hline${ }^{\text {a}}$ Pre-hospital delay time, hours & $3.66 \pm 5.15$ & $4.46 \pm 10.97$ & -1.618 & 0.106 \\
\hline Door-to-balloon time, $\min$ & $91.26 \pm 137.56$ & $95.27 \pm 50.50$ & -0.461 & 0.645 \\
\hline${ }^{\mathrm{b}}$ Killip classification & $1.27 \pm 0.74$ & $\mathrm{I} .44 \pm 0.83$ & -1.917 & 0.056 \\
\hline Number of diseased vessels & $1.58 \pm 0.63$ & $1.85 \pm 0.72$ & -3.465 & 0.001 \\
\hline Arrhythmia during $\mathrm{PCl}, \mathrm{n}(\%)$ & $6(5.8 \%)$ & $73(19.8 \%)$ & 11.453 & 0.001 \\
\hline Complications during $\mathrm{PCl}, \mathrm{n}(\%)$ & $3(2.9 \%)$ & $25(6.8 \%)$ & 2.205 & 0.138 \\
\hline CK-MB, $\mathrm{mmol} / \mathrm{L}$ & $109.13 \pm 127.16$ & $113.16 \pm 128.25$ & -0.284 & 0.777 \\
\hline $\mathrm{TnT}, \mathrm{ng} / \mathrm{L}$ & $4.07 \pm 3.96$ & $4.42 \pm 3.77$ & -0.792 & 0.429 \\
\hline MACEs at 6-month, $\mathrm{n}(\%)$ & $4(3.8 \%)$ & $74(20.1 \%)$ & 25.495 & 0.000 \\
\hline
\end{tabular}

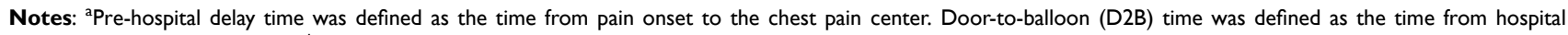

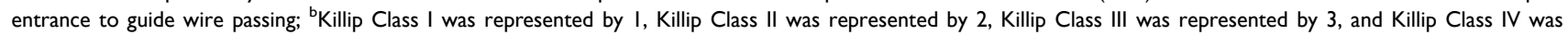
represented by 4 .

Abbreviations: FHPCHD, family history of premature coronary heart disease; HMD, History of myocardial infarction; BMI, body mass index; PCl, percutaneous coronary intervention; CK-MB, creatine kinase myocardial band; TnT, Troponin T; MACEs, major adverse cardiac events.

$\mathrm{p}=0.01)$ and significant improvement in 6-MWD (OR 7.999, 95\% CI $4.342-14.737, \mathrm{P}=0.00)$ and LVEF (OR $1.112,95 \%$ CI $1.072-1.154, \mathrm{p}=0.00$ ).

Multivariate analysis of MACEs (Table 4) showed that there were 6 factors were associated with the occurrence of MACEs, they were age $>65$ years (OR 1.032 , 95\% CI 1.009$1.009, \mathrm{p}=0.007$ ), smoking history (OR 0.485, 95\% CI $0.238-$ $0.989, \mathrm{p}=0.046$ ), education level (OR 2.646, 95\% CI 1.370 5.108, $\mathrm{p}=0.004$ ), 6-MWD (OR 1.688, 95\% CI 1.104-2.811, $\mathrm{p}=0.044)$, LVEF (OR 0.958, 95\% CI 0.926-0.991, $\mathrm{p}=0.013)$ and CR (OR 6.271, 95\% CI 2.236-17.590, p=0.000).

\section{Discussion}

There is an increasing number of STEMI patients after primary PCI who receive CR including exercise rehabilitation in China, the proportion group $\mathrm{A}$ in our study was $22.0 \%$. Our study showed that comprehensive rehabilitation treatment significantly reduced the incidence of

Table 2 ANCOVA Analysis 4 Indicators Before and After CR

\begin{tabular}{|l|c|c|c|c|c|c|}
\hline \multirow{2}{*}{ Indicators } & \multicolumn{2}{|c|}{ Group A } & \multicolumn{2}{c|}{ Group B } & \multirow{2}{*}{ F } \\
\cline { 2 - 6 } & Within 24 Hours & 6 Month Later & Within 24 Hours & 6 Month Later & \\
\hline NT-proBNP (pg/L) & $1456.35 \pm 1071.28$ & $155.01 \pm 162.05$ & $2400.25 \pm 3254.32$ & $931.45 \pm 1683.00$ & 1.427 & 0.027 \\
6-MWD (meter) & $312.56 \pm 16.71$ & $632.43 \pm 10.89$ & $320.08 \pm 15.69$ & $486.52 \pm 16.07$ & 51.72 & 0.000 \\
LVEF(\%) & $56.55 \pm 6.57$ & $60.73 \pm 6.17$ & $54.42 \pm 8.21$ & $54.68 \pm 9.37$ & 2.136 & 0.000 \\
SWMA, n(\%) & $73(70.2 \%)$ & $69(66.3 \%)$ & $292(79.1 \%)$ & $262(71.0 \%)$ & 0.025 & 0.875 \\
\hline
\end{tabular}

Abbreviations: NT-proBNP, N-terminal pro-B-type natriuretic peptide; 6-MWD, 6-minute walk distance; LVEF, Left ventricular ejection fraction; SWMA, Segmental wall motion abnormalities. 
Table 3 Results of Multivariate Logistic Regression Analysis for CR (McFadden's Rho-Squared $=0.715$ )

\begin{tabular}{|l|l|l|l|}
\hline Variables & OR & 95\% CI & P \\
\hline Age>65 & 0.917 & $0.859-0.979$ & 0.009 \\
Gender & 2.253 & $0.475-10.694$ & 0.306 \\
Hyperlipidemia & 3.060 & $1.132-8.274$ & 0.028 \\
FHPCHD & 2.436 & $0.225-26.389$ & 0.464 \\
Smoking history & 0.508 & $0.171-1.512$ & 0.224 \\
Higher education & 0.014 & $0.003-0.060$ & 0.000 \\
BMI (kg/m ${ }^{2}$ ) & 0.931 & $0.710-1.221$ & 0.606 \\
Heart rate, bpm & 1.001 & $0.975-1.027$ & 0.969 \\
Number of diseased vessels & 0.778 & $0.409-1.479$ & 0.444 \\
Arrhythmia during PCI & 0.286 & $0.071-1.147$ & 0.077 \\
NT-proBNP & 1.000 & $0.999-1.000$ & 0.168 \\
6-MWD (meter) & 0.009 & $0.002-0.044$ & 0.000 \\
LVEF (\%) & 0.930 & $0.816-1.059$ & 0.271 \\
NT-proBNP at 6-month & 0.998 & $0.997-1.000$ & 0.000 \\
6-MWD (meter) at 6-month & 7.999 & $4.342-14.737$ & 0.000 \\
LVEF (\%) <54.68 9.37 & 1.112 & $1.072-1.154$ & 0.000 \\
SWMA & 1.278 & $0.802-2.036$ & 0.302 \\
MACEs at 6-month follow-up & 0.191 & $0.038-0.961$ & 0.045 \\
\hline
\end{tabular}

Abbreviations: OR, odds ratio; $\mathrm{Cl}$, confidence interval; $\mathrm{FHPCHD}$, family history of premature coronary heart disease; $\mathrm{BMI}$, body mass index; $\mathrm{PCl}$, percutaneous coronary intervention; NT-proBNP, N-terminal pro-B-type natriuretic peptide; 6-MWD, 6-minute walk distance; LVEF, Left ventricular ejection fraction; SWMA, Segmental wall motion abnormalities; MACEs, major adverse cardiac events.

MACEs during 6-month follow-up in patients with STEMI after PCI. The rate of MACES of patients treated by CR was 5 times lower than the patients without CR even they had much higher rates of hyperlipidemia. Patients' low mean age and higher LVEF at 6 months may be the favorable factors contributing to the low MACEs. CR and education were associated with lower MACEs in patients with acute coronary syndrome. ${ }^{14}$

ANCOVA analysis showed that after CR, the NTproBNP levels, 6-MWD and LVEF were significantly improved when compared group A to group B. Further analysis indicated that 6 -MWD at 6 -month was about 8 times better in group A than in group B.

The fact that CR reduces the incidence of MACEs and restenosis is due to improvements in blood rheology and endothelial function. ${ }^{15}$ Vascular endothelium is the primary regulator of blood flow through its vasodilatory properties. This is primarily through the release of nitric oxide (NO), but also prostacyclin (PGI2) and endothelialderived hyperpolarizing factor (EDHF), in response to various chemicals (eg, 5-hydroxytryptamine, bradykinin and acetylcholine), and physical stimuli (eg, shear stress). ${ }^{16}$ Exercise training augments the expression of NO synthase in endothelial cells and induces the up-
Table 4 Results of Multivariate Logistic Regression Analysis for MACEs (McFadden's Rho-Squared = 0.083)

\begin{tabular}{|l|l|l|l|}
\hline Variables & OR & $\mathbf{9 5 \%}$ CI & P \\
\hline Age>65 & 1.032 & $1.009-1.005$ & 0.007 \\
Gender & 0.922 & $0.475-10.694$ & 0.794 \\
Hyperlipidemia & 1.233 & $0.745-2.007$ & 0.426 \\
FHPCHD & 0.824 & $0.174-3.909$ & 0.807 \\
Smoking history & 0.485 & $0.238-0.989$ & 0.046 \\
Higher education & 2.646 & $1.370-5.108$ & 0.004 \\
BMI (kg/m ${ }^{2}$ ) & 0.944 & $0.849-1.049$ & 0.282 \\
Heart rate, bpm & 0.997 & $0.983-1.012$ & 0.726 \\
Number of diseased vessels & 1.167 & $0.829-1.644$ & 0.377 \\
Arrhythmia during PCI & 1.315 & $0.709-2.439$ & 0.384 \\
NT-proBNP & 1.000 & $1.000-1.000$ & 0.199 \\
6-MWD (meter) & 1.688 & $1.014-2.811$ & 0.044 \\
LVEF (\%) & 0.958 & $0.926-0.991$ & 0.013 \\
NT-proBNP at 6-month & 1.000 & $1.000-1.000$ & 0.488 \\
6-MWD (meter) at 6-month & 0.596 & $0.343-1.037$ & 0.067 \\
LVEF (\%) <54.68 9.37 & 1.009 & $0.968-1.052$ & 0.669 \\
SWMA & 1.342 & $0766-2.351$ & 0.304 \\
CR & 6.271 & $2.236-17.590$ & 0.000 \\
\hline
\end{tabular}

Abbreviations: $\mathrm{OR}$, odds ratio; $\mathrm{Cl}$, confidence interval; $\mathrm{FHPCHD}$, family history of premature coronary heart disease; $\mathrm{BMI}$, body mass index; $\mathrm{PCl}$, percutaneous coronary intervention; NT-proBNP, N-terminal pro-B-type natriuretic peptide; 6-MWD, 6-minute walk distance; LVEF, Left ventricular ejection fraction; SWMA, Segmental wall motion abnormalities; CR, Cardiac rehabilitation.

regulation of copper-and-zinc-containing superoxide dismutase in the cytosol. ${ }^{17,18}$ Exercise rehabilitation can increase cardiac blood flow, and increase myocardial contractility, and improve the heart pumping strength. ${ }^{19}$ Exercise training has been shown to attenuate ST depression during exercise and reduce hypoperfusion on thallium scanning, suggesting that myocardial perfusion may increase. ${ }^{19}$

In addition, exercise rehabilitation can enhance parasympathetic tone, ${ }^{20}$ alter the production of angiotensin II and reduce aldosterone production, thereby reducing sympathetic tone, and complementing the effects of other exercise-induced modulators of parasympathetic activity such as plasma adrenomedullin and atrio/brain-natriureticpeptides. ${ }^{21,22}$ These effects are related to aerobic capacity and work by inhibiting endothelin-1 and norepinephrine, leading to improved endothelial function and responsiveness. $^{23,24}$

A recent retrospective analysis conducted in Korea found that CR treatment was accompanied by lower MACE, especially in patients with low pre-procedure TIMI blood flow in primary PCI for STEMI. ${ }^{7}$ Our results on the relationship between CR and MACEs were consistent with those reported in Korea. In addition, several 
studies investigated that early and regular CR may reduce the incidence of lumen loss, coronary restenosis, and vascular events after stenting after PCI in patients with AMI patients and promote early physical recovery and improve quality of life. $^{25,26} \mathrm{~A}$ meta-analysis of the CR effect on left ventricular remodeling after MI showed that the earlier and longer the exercise training, the more significant the reversal cardiac remodeling. ${ }^{27}$

According to our results, higher NT-proBNP, lower LVEF and the shorter 6-MWD had a statistically significant impact on MACEs in patients with STEMI after primary PCI. Patients with reduced LVEF have more potential risk factors and an increased incidence of thromboembolic events, which may contribute to the increased cardiovascular mortality. $^{28}$ Results from the Australian Capital Territory PCI Registry showed that the incidence of MACEs was strongly associated with door-to-balloon time, which was an independent predictor of MACEs. ${ }^{29}$ The prolonged ischemia, increased oxidative stress, and increased MI size will increase the risk of MACEs. $^{29}$

Our results from this study have valuable clinical implications for STEMI patients accepting primary PCI. CR significantly reduced the incidence of MACEs during 6-month follow-up in STEMI patients after PCI. Delayed CR after myocardial infarction may lead to a reduced quality of life after MI. In patients with AMI, an early home-based CR program can greatly improve ventricular function in the short term. ${ }^{30}$ Long et al found that CR may have little effect or no difference on all-cause mortality in the short term. ${ }^{31}$ In our study, the incidence of recurrent angina was significantly lower in group A than in group $B$ and no patients died in either group, which may be related to the short duration of follow-up. Thus, it may be safe and effective to start CR on day 10 on average for STEMI patients after primary PCI.

Despite the well-known benefits of CR, adherence to CR remains low. ${ }^{32}$ In the United States and Europe, CR is performed in less than $30 \%$ of patients with indications for CR. ${ }^{13,33}$ And the percentage of Asian patients participating in CR after PCI was lower than that reported in western countries. We hope that our study can encourage more patients to participate in $\mathrm{CR}$.

The limitations of this study are as follows: (1) only patients from one tertiary grade A hospital in China were included; and (2) some potential relative factors such as socioeconomic situation, basic medical insurance and social support were not collected. (3) Additional research is needed to improve our understanding of the relationship between $\mathrm{CR}$ and long-term clinical prognosis.

\section{Conclusion}

CR significantly reduces NT-proBNP levels and significantly improves 6-MWD and LVEF, thereby reducing MACEs in STEMI patients after PCI. CR including exercise rehabilitation is a useful option for reduced MACEs in STEMI patients after primary PCI.

\section{Data Sharing Statement}

The analyzed data sets generated during the study are available from the corresponding authors on reasonable request.

\section{Ethics Approval and Consent to Participate}

The study was proceeded conforming to the ethical guidelines of the Declarations of Helsinki. The study was approved by The Second Affiliated Hospital of Shenyang Medical College. All participants had signed the written consent forms.

\section{Funding}

This study was funded by Key R \& D fund of Liaoning Provincial Department of Science and Technology (2018225063).

\section{Disclosure}

The authors report no conflicts of interest in this work.

\section{References}

1. Bäck M, Caldenius V, Svensson L, et al. Perceptions of kinesiophobia in relation to physical activity and exercise after myocardial infarction: a qualitative study. Phys Ther. 2020;100:2110-2119. doi:10.1093/ptj/ pzaa159

2. Vogel B, Claessen BE, Arnold SV, et al. ST-segment elevation myocardial infarction. Nat Rev Dis Primers. 2019;5(1):39. doi:10.1038/ s41572-019-0090-3

3. Fazel R, Joseph TI, Sankardas MA, et al. Comparison of reperfusion strategies for ST-segment-elevation myocardial infarction: a multivariate network meta-analysis. $J$ Am Heart Assoc. 2020;9(12): e015186. doi:10.1161/JAHA.119.015186

4. Hu SS, Gao RL, Liu LS, et al. Summary of the 2018 report on cardiovascular diseases in China. Chin Circ J. 2019;34(3):209-220.

5. Zhao W, Bai J, Zhang F, et al. Impact of completeness of revascularization by coronary intervention on exercise capacity early after acute ST-elevation myocardial infarction. J Cardiothorac Surg. 2014;9:50. doi:10.1186/1749-8090-9-50

6. Bhagwat MM, Woods JA, Dronavalli M, et al. Evidence-based interventions in primary care following acute coronary syndrome in Australia and New Zealand: a systematic scoping review. BMC Cardiovasc Disord. 2016;16(1):214. doi:10.1186/s12872-016-0388-y 
7. Lee HY, Hong SJ, Jung IH, et al. Effect of cardiac rehabilitation on outcomes in patients with ST-elevation myocardial infarction. Yonsei Med J. 2019;60(6):535-541. doi:10.3349/ymj.2019.60.6.535

8. Wenger NK. Current status of cardiac rehabilitation. J Am Coll Cardiol. 2008;5:1619-1631. doi:10.1016/j.jacc.2008.01.030

9. Bellmann B, Lin T, Greissinger K, et al. The beneficial effects of cardiac rehabilitation. Cardiol Ther. 2020;9:35-44. doi:10.1007/ s40119-020-00164-9

10. Poh R, Ng HN, Loo G, et al. Cardiac rehabilitation after percutaneous coronary intervention in a multiethnic Asian country: enrollment and barriers. Arch Phys Med Rehabil. 2015;96(9):1733-1738. doi:10.1016/j.apmr.2015.05.020

11. Chinese Society of Cardiology of Chinese Medical Association, Editorial Board of Chinese Journal of Cardiology. 2019 Chinese Society of Cardiology (CSC) guidelines for the diagnosis and management of patients with ST-segment elevation myocardial infarction. Chin J Cardiol. 2019;47(10):766-783. doi:10.3760/cma.j.issn.02533758.2019.10.003

12. Goel K, Pack QR, Lahr B, et al. CR is associated with reduced long-term mortality in patients undergoing combined heart valve and coronary artery bypass graft (CABG) surgery. Eur J Prev Cardiol. 2015;22:159-168. doi:10.1177/2047487313512219

13. Chinese Society of Cardiology of Chinese Medical Association, Cardiovascular Committee of Chinese Association of Rehabilitation Medicine, Cardiocerebral Vascular Disease Professional Committee of Chinese Association of Gerontology. Chinese expert consensus on the rehabilitation and secondary prevention of coronary heart disease. Chin J Cardiol. 2013;41(4):267-275. doi:10.3760/cma.j.issn.02533758.2013.04.003

14. Al-Zakwani I, Mabry RM, Zubaid M, et al. Association between education and major adverse cardiac events among patients with acute coronary syndrome in the Arabian Gulf. BMJ Glob Health. 2019;4(1):e001278. doi:10.1136/bmjgh-2018-001278

15. Dendale P, Berger J, Hansen D, et al. Cardiac rehabilitation reduces the rate of major adverse cardiac events after percutaneous coronary intervention. Eur J Cardiovasc Nurs. 2005;4(2):113-116. doi:10.1016/j.ejcnurse.2004.11.003

16. Lanza GA, Golino M, Villano A, et al. Cardiac rehabilitation and endothelial function. J Clin Med. 2020;9(8):2487. doi:10.3390/ jcm 9082487

17. Paul JD, Powell TM, Thompson M, et al. Endothelial progenitor cell mobilization and increased intravascular nitric oxide in patients undergoing cardiac rehabilitation. J Cardiopulm Rehabil Prev. 2007;27(2):65-73. doi:10.1097/01.HCR.0000265031.10145.50

18. Belardinelli R. Esercizio fisico e funzione endoteliale in pazienti coronaropatici [Effect of exercise on coronary endothelial function in patients with coronary artery disease]. Ital Heart J Suppl. 20001;1 (7):945-946.

19. Yang X, Li Y, Ren X, et al. Effects of exercise-based cardiac rehabilitation in patients after percutaneous coronary intervention: a meta-analysis of randomized controlled trials. Sci Rep. 2017;7:44789. doi:10.1038/srep44789

20. Kachur S, Lavie CJ, Morera R, et al. Exercise training and cardiac rehabilitation in cardiovascular disease. Expert Rev Cardiovasc Ther. 2019;17(8):585-596. doi:10.1080/14779072.2019.1651198
21. Kalupahana NS, Moustaid-Moussa N. The adipose tissue renin-angiotensin system and metabolic disorders: a review of molecular mechanisms. Crit Rev Biochem Mol Biol. 2012;47(4):379-390. doi:10.3109/10409238.2012.694843

22. Daniłowicz-Szymanowicz L, Figura-Chmielewska M, Ratkowski W, et al. Effect of various forms of physical training on the autonomic nervous system activity in patients with acute myocardial infarction. Kardiol Pol. 2013;71(6):558-565. doi:10.5603/KP.2013.0118

23. Kachur S, Chongthammakun V, Lavie CJ, et al. Impact of cardiac rehabilitation and exercise training programs in coronary heart disease. Prog Cardiovasc Dis. 2017;60(1):103-114. doi:10.1016/j. pcad.2017.07.002

24. Smart NA, Meyer T, Butterfield JA, et al. Individual patient meta-analysis of exercise training effects on systemic brain natriuretic peptide expression in heart failure. Eur J Prev Cardiol. 2012;19 (3):428-435. doi:10.1177/1741826711409171

25. Munk PS, Breland UM, Aukrust P, et al. High intensity interval training reduces systemic inflammation in post-PCI patients. Eur J Cardiovasc Prev Rehabil. 2011;18(6):850-857. doi:10.1177/ 1741826710397600

26. Lee HY, Kim JH, Kim BO, et al. Regular exercise training reduces coronary restenosis after percutaneous coronary intervention in patients with acute myocardial infarction. Int $J$ Cardiol. 2013;167 (6):2617-2622. doi:10.1016/j.ijcard.2012.06.122

27. Haykowsky M, Scott J, Esch B, et al. A meta-analysis of the effects of exercise training on left ventricular remodeling following myocardial infarction: start early and go longer for greatest exercise benefits on remodeling. Trials. 2011;12:92. doi:10.1186/1745-6215-12-92

28. Lee JY, Sunwoo JS, Kwon KY, et al. Left ventricular ejection fraction predicts poststroke cardiovascular events and mortality in patients without atrial fibrillation and coronary heart disease. Korean Circ J. 2018;48(12):1148-1156. doi:10.4070/kcj.2018.0115

29. Chandrasekhar J, Marley P, Allada C, et al. Symptom-to-balloon time is a strong predictor of adverse events following primary percutaneous coronary intervention: results from the australian capital territory PCI registry. Heart Lung Circ. 2017;26(1):41-48. doi:10.1016/j. hlc.2016.05.114

30. Xu L, Cai Z, Xiong M, et al. Efficacy of an early home-based cardiac rehabilitation program for patients after acute myocardial infarction: a three-dimensional speckle tracking echocardiography randomized trial. Medicine. 2016;95(52):e5638. doi:10.1097/ MD.0000000000005638

31. Long L, Mordi IR, Bridges C, et al. Exercise-based cardiac rehabilitation for adults with heart failure. Cochrane Database Syst Rev. 2019;1(1):CD003331. doi:10.1002/14651858.CD003331

32. Way KL, Vidal-Almela S, Keast ML, et al. The feasibility of implementing high-intensity interval training in cardiac rehabilitation settings: a retrospective analysis. BMC Sports Sci Med Rehabil. 2020;12:38. doi:10.1186/s13102-020-00186-9

33. Ades PA, Keteyian SJ, Wright JS, et al. Increasing cardiac rehabilitation participation from $20 \%$ to $70 \%$ : a road map from the million hearts cardiac rehabilitation collaborative. Mayo Clin Proc. 2017;92 (2):234-242. doi:10.1016/j.mayocp.2016.10.014
International Journal of General Medicine

\section{Publish your work in this journal}

The International Journal of General Medicine is an international, peer-reviewed open-access journal that focuses on general and internal medicine, pathogenesis, epidemiology, diagnosis, monitoring and treatment protocols. The journal is characterized by the rapid reporting of reviews, original research and clinical studies
Dovepress

across all disease areas. The manuscript management system is completely online and includes a very quick and fair peer-review system, which is all easy to use. Visit http://www.dovepress.com/ testimonials.php to read real quotes from published authors. 